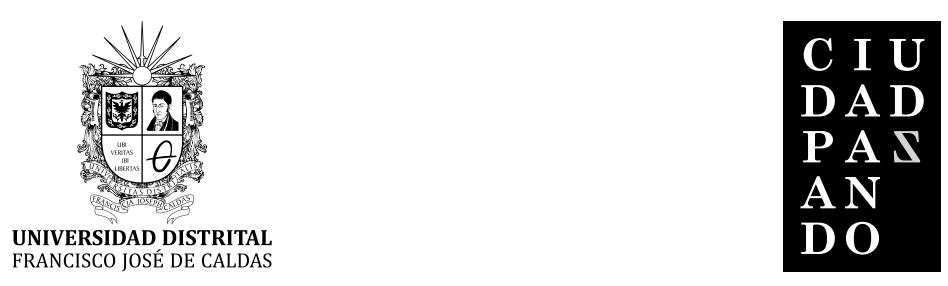

\title{
El triunfo del No: la paradoja emocional detrás del plebiscito
}

\section{Mauricio Hernández Pérez ${ }^{1}$}

Para citar este artículo: Hernández, M. (2017). El triunfo del No: la paradoja emocional detrás del plebiscito. Revista Ciudad Paz-ando, 10(2), 92-96.

doi: https://doi.org/10.14483/2422278X.12218

Fecha de recepción: 16 de junio de 2017

Fecha de aprobación: 31 de octubre de 2017

\section{¿Por qué el no? ¡Porque sí... y punto!}

Los resultados del plebiscito refrendatorio realizado el 2 de octubre de 2016 y presentados por la Registraduría Nacional del Estado Civil fueron más que contundentes: de un potencial electoral de 34899945 de personas en Colombia, apenas 13066047 fueron quienes se acercaron a las mesas para definir la aceptación (o no) de lo acordado en su momento. De estas, 6431376 votaron por el no (50.21\%), mientras que por el sí lo hicieron 6377482 (el $49,78 \%$ ). La diferencia entre ambos resultados fue de apenas 53894 votos, una cifra muy pequeña pero con grandes consecuencias de lo que fue y de lo que será el futuro del país. Pero, como se ha vuelto una costumbre dentro de la cultura política colombiana, la gran vencedora fue la abstención, pues no ejercieron este derecho 21833898 personas (es decir, el 62.5\% del censo posible de votantes).

Las reacciones ante la situación no se hicieron esperar y los medios, más por el afán de comunicar los resultados, dieron rienda suelta a la ya acostumbrada "pirotecnia mediática" que se hizo manifiesta en algunos titulares: "Polarización del país, reflejada en resultados del escrutinio" (El Tiempo), "Resultados del plebiscito: el No se impuso en la jornada electoral" (Semana), "Dólar sube por resultado del plebiscito que rechazó el Acuerdo de Paz" (Dinero), "Así registra el mundo el triunfo del NO en el plebiscito en Colombia" (El Espectador).

Con el tiempo, se abrieron paso a las columnas de opinión y análisis en un intento por ahondar en los elementos explicativos del asunto: "Las molestas verdades que dejó el 'mapa del plebiscito’” (El País), “¿Dónde está la gente de la paz?" (Revista Arcadia), "El triunfo del NO y el fracaso de la educación colombiana” (Semana).

Bajo esta dinámica de incertidumbre y expectativa que en su momento se dio (y que aún hoy continúa siendo motivo de preocupación), asalta la duda sobre cuál es la reflexión que, desde la academia, podría hacerse sobre este fenómeno y resultado en particular. Es aquí donde el texto que se reseña, escrito por Andrei Gómez-Suárez, doctor en Relaciones Internacionales y magíster en Guerras Contemporáneas y Estudios de Paz de la Universidad de Sussex, especialista en Resolución de conflictos armados y politólogo de la Universidad de los Andes de Bogotá D.C., ocupa y tiene pertinencia, toda vez que se constituye como una de las primeras (y esperemos que no la única) aproximación realizada sobre el fenómeno desde la academia.

\footnotetext{
1 Magíster en Estudios Políticos, Universidad Nacional de Colombia. Docente del Programa de Negocios y Relaciones Internacionales, Universi-
} dad de La Salle, Colombia. Correo electrónico: mahernandezpe@gmail.com 
Podríamos señalar, junto con Jaramillo (2014), que el papel de Gómez-Suárez se acerca más al de un "activista teórico", es decir, aquel quien siente tener un fuerte compromiso ético para con la sociedad en general y quien, con rigor académico, acompaña sus reflexiones a través del impulso de iniciativas como Rodeemos el diálogo (ReD) experiencia que, como es presentada en su página web, constituye:

una red transnacional, no partidista, de colombianos y amigos de Colombia que surgió en el Reino Unido entre septiembre y octubre de 2012, cuando iniciaron los diálogos de paz entre el gobierno Santos y las Farc. ReD apoya la solución negociada de conflictos armados y aporta a la construcción de paz mediante el fortalecimiento de una cultura de diálogo. En 2017, ReD tiene tres objetivos: (1) acompañar la implementación del acuerdo de paz Gobierno-Farc, (2) apoyar la negociación Gobierno-ELN y (3) reflexionar en pos de la reconciliación con diversos sectores. ReD cree en el poder de un diálogo basado en seis principios: el respeto para escuchar y valorar la diferencia, la generosidad para compartir, la honestidad para reconstruir la confianza, la solidaridad para ponerse en el lugar de otros, la corresponsabilidad para construir desde la diversidad y la Autocrítica para revaluar y crecer. ReD construye alianzas con universidades y organizaciones gubernamentales y no gubernamentales, movimientos sociales y personalidades públicas- para integrarlas en una expresión de ciudadanía activa. (ReD, s.f.).

A través de lo que se ha denominado como una cultura de diálogo, Rodeemos el diálogo a través de conversatorios, desayunos de paz, tertulias de reconciliación y los "No-talleres", se constituyó como una de las tantas plataformas colaborativas cuyo propósito era realizar una pedagogía en construcción de paz.

En este orden de ideas, el texto que se reseña a continuación adquiere una particular vigencia; para el "calor" del momento se constituyó como uno de los escritos que procuró respuestas y reflexiones en frío, ya no desde la perspectiva de la pirotecnia mediática o de lo que columnistas de opinión presentaron de manera casi que inmediata aunque sí tomando como referente lo que, en su momento, desde los medios se canalizó hacia la opinión pública ${ }^{2}$.

2 Aunque como el autor mismo indica en la introducción a su texto, hay tres salvedades metodológicas que se requieren señalar (de las cuales solo destacamos la primera): "En primer lugar, la reconstrucción de la estrategia uribista se basa fundamentalmente en revisión de prensa, en particular de los periódicos El Espectador y El Tiempo de cobertura nacional, así como de la revista Semana. Por tanto, deja por fuera del análisis los espacios de RCN y Caracol, canales televisivos que han
De manera más reciente - y posterior a la publicación del texto de Gómez-Suárez-, De Miera (2017) desarrolla en su trabajo una reflexión sobre el papel de la sociedad civil colombiana y el comportamiento de esta durante los resultados del plebiscito. Lo particular de este texto es que ofrece una mirada externa, particularmente desde España — de hecho, es llamativo como titula la primera parte de su texto: "uno que viene de fuera" y la última: "palabras desde España"-, ameritaría una posterior reseña ante la ausencia de material académico conducente hacia la identificación de explicaciones plausibles sobre lo ocurrido. Llama la atención que Gómez-Suárez y De Miera coincidan en una idea central para explicar parte de los resultados del no, esto es, el fortalecimiento de un sentimiento generalizado: el miedo. Podría decirse que el miedo tramitado en una doble vía: el miedo top-down, es decir, aquel gestado desde las fuerzas políticas hacia el conjunto y grueso de la sociedad civil, pero también el miedo down-top, infundado por quienes desinformados - y creyendo en la desinformación- replicaron el mensaje hacia el grueso de la sociedad colombiana.

Pero ¿miedo a qué?:

miedo a la implementación de un delirante castro-chavismo, miedo a la pérdida de prebendas y privilegios, miedo a la pérdida del concepto de propiedad privada. Precisamente, tal como enseña la historia, los miedos más paralizadores y efectivos entre los que menos tienen. Entiendo que fue así, en gran medida, como se gestó la pinza del No, desde arriba y desde debajo de la sociedad colombiana. (De Miera, 2017, p. 62)

Como sea, la producción académica sobre el fenómeno está por producirse, pero contar con unas primeras aproximaciones contribuye a cualificar la reflexión que, hacia el futuro, se desarrolle, y para esto, dar cuenta de una de las primeras aproximaciones que se dio sobre el tema se hace mucho más que útil.

En este orden de ideas, la reseña presenta tres momentos: el primero (las audiencias) en el que se describen los grupos poblacionales hacia quienes el texto se encuentra dirigido; un segundo momento corresponde a una reconstrucción, si no en detalle sí por lo menos en términos, de ideas fuerza del texto; por último, se presentan algunas ideas de reflexión derivadas por parte del autor.

contribuido con fuerza al establecimiento de las narrativas uribistas" (Gómez, 2016, p.22). 


\section{Las audiencias ${ }^{3}$}

Lo primero que llama la atención del texto y que se ve reflejado en la introducción, lo cual podría interpretarse a su vez como un criterio pedagógico seguido por parte del autor o, quizás, una señal de respeto para quien se introduce en sus líneas, tiene que ver con las audiencias hacia las cuales está dirigido el ensayo, elemento que habitualmente se deja de lado por parte de quienes presentan desarrollos de orden reflexivo o teórico.

En este orden de ideas, los miembros y simpatizantes del Centro Democrático se presentan como la primera audiencia, esto con un firme propósito: incorporar elementos que posibiliten ciertas dosis de autocrítica a la colectividad (ejercicio aún en deuda por parte de quienes la conforman); en segundo lugar, se encuentran los integrantes del gobierno, así como de las organizaciones sociales, partidos políticos, gremios y ciudadanos que apoyaron el sí; en tercer lugar, los ciudadanos indiferentes, aquellos sobre quienes no sabemos si continúan aún hoy día manteniéndose como indiferentes ante las realidades que nos ofrece el país y, por último, pero no menos importantes los jóvenes, quienes tuvieron (y continúan teniendo) una gran responsabilidad hacia futuro.

Se podría considerar como un acierto esta primera parte porque quien se aproxime al texto podría "hacerse el autoexamen" y eventualmente reconocerse como parte de algunas de estas audiencias para, en este orden de ideas, asumir las consideraciones a las que hubiese lugar; sin embargo, también habría otro escenario, que quien se acercara por primera vez al texto no se reconozca como parte de ninguna de las audiencias que el autor menciona, lo que haría más rico el alcance del texto en lo que a esta materia corresponde, pues quedaría manifiesto que el ejercicio planteado - en términos de audiencias- es incompleto y cabría la posibilidad de seguir indagando al respecto.

\section{El texto}

Con respecto al problema central, objeto de preocupación, pregunta de investigación o cualquier otro apelativo con el que se quiera acercarse a la intensión del autor al momento de escribir este ensayo, un conjunto de referentes distribuidos a lo largo de la introducción da cuenta de ello y gozan de un "enganche" atractivo en función de cómo son presentados.

Se hace necesario señalar que el texto se propone una mirada crítica a la cartografía emocional de lo que sucedió para alimentar los resultados del plebiscito. El texto

3 Se hace necesario destacar que algunos de los elementos dispuestos en esta reseña derivan de los aportes presentados en un conversatorio con el autor del libro realizado el 23 de mayo de 2017 por parte del Instituto de Estudios Políticos y Relaciones Internacionales (Iepri) de la Universidad Nacional de Colombia en Bogotá. se propone, además, hacer un diagnóstico, ir un poco más allá, y procurar un ejercicio que permita "mapear la esperanza" desde la perspectiva afectiva emocional del momento transicional de Colombia y que permitiría tramitar emocionalmente la transición de la guerra hacia la paz. En términos mucho más concretos, el autor se propone examinar el marco de referencia emocional adverso al proceso de paz en un intento por dar respuesta a la pregunta ¿cómo logró el uribismo el triunfo del no? De esta pregunta central se desprenden otras subsidiarias y no menos importantes: ¿cuál es el componente emocional que explican los resultados del plebiscito?, y ¿a qué estrategias emocionales se recurrieron?

Las inquietudes y preocupaciones anteriormente planteadas tienen como fundamento un punto que se materializó en los resultados del 2 de octubre de 2016: "La gente no se conectó". Ante esta problemática, por lo pronto tres razones parecerían alimentar una explicación plausible en la totalidad del ensayo: (a) emocionalmente la gente no comprendió la importancia de ir a las urnas, (b) existe un descrédito e incredulidad hacia la política y (c) no es claro aún determinar con exactitud cómo se posicionan los actores políticos.

El texto se encuentra dividido en cuatro partes y un apartado de reflexiones finales. La primera parte ("De la ruptura Santos-Uribe a la oposición del Centro Democrático"), Gómez-Suárez establece que lo acontecido el 2 de octubre se explica en relación con un conjunto de emociones fundamentales (no es gratuito que el subtítulo del texto intente hacer el llamado de atención sobre esto). Destaca la génesis de la cuestión: la traición que Uribe sintió por parte de Santos a medida que avanzaba su mandato y que trajo consigo un elemento polarizador determinante. La aceptación de un conflicto armado por parte de Juan Manuel Santos y que se constituyó como ventana de oportunidad para la apertura del proceso de paz fue, entre otros factores, ejemplo de ello. Como sea, la reacción emocional de Uribe no fue algo que le afectó solamente a él como persona, como sujeto, sino que fue una sensación que en persona logró tramitar a través de la bancada y los simpatizantes del Centro Democrático hacia el resto del país y que trajo consigo una polarización de este a través de un marco de referencia emocional adverso al proceso de paz (idea central trabajada por Gómez-Suárez a lo largo de su texto), y que posplebiscito ha quedado sembrado como una herencia para el futuro del país pero que se ha constituido a la par como "un trauma que heredamos del nacimiento de la República: la negación del otro a través de narrativas que simplifican la realidad y justifican la violencia para alcanzar un fin" (p. 36).

El segundo apartado ("El arsenal discursivo del Centro Democrático") desarrolla una cartografía de lo que el autor ha denominado como el arsenal de dispositivos 
retóricos, acuñados durante cinco años, específicamente entre septiembre de 2011 y agosto de 2016 como antesala del ataque frontal al proceso de refrendación del acuerdo final de paz, a través de un conjunto de acciones y reacciones por parte de un proyecto político de oposición. Valdría la pena señalar que, si bien la categoría de arsenal de dispositivos retóricos, entendidos como términos, narrativas y mecanismos a través de los cuales se conectan hechos políticos con la emocionalidad de los colombianos con el fin de hacer contrapeso al proyecto político de Santos, es un elemento que llama la atención dentro de la totalidad del texto, particularmente del segundo apartado, no solo por el juicioso y sistemático examen, recuento y análisis de prensa que se hace de cada uno de los seis dispositivos considerados -el castrochavismo, paz sin impunidad, Santos entrega Colombia a las FARC, no más Santos, resistencia civil, y la ideología de género-, sino porque es una categoría que por el excesivo uso dado por el autor, puede llegar a producir una sensación al lector de agotamiento y sobrevaloración. En definitiva, cuando una idea es reiterada de manera excesiva, su fuerza, de alguna $\mathrm{u}$ otra manera, tiende a perderse.

Como sea, y aunque criticable en términos operativos, la idea del arsenal de dispositivos retóricos es uno de los aportes que el autor apuntala en su ensayo toda vez que es desde allí donde se podría explicar la manipulación emocional que trajo consigo la férrea oposición al proceso de paz y los resultados obtenidos; básicamente aquí se aventura una de las ideas explicativas de los resultados del 2 de octubre: esto fue posible en tanto rabia, miedo, decepción e indignación frente al proceso de paz se constituyeron como las emociones que fueron tramitadas y movilizadas dentro del pleno de la sociedad colombiana desde el uribismo.

La tercera parte del ensayo ("La campaña por el No del Centro Democrático"), mucho más descriptiva, da cuenta de la dinámica y estrategia que los simpatizantes del Centro Democrático emplearon en su contraofensiva y campaña por el no. La estrategia, para el autor, fue básicamente "sacar a la gente a votar verraca" (Gómez-Suárez, 2016, p.72), y ello solo fue posible a través de la tramitación y reiteración, que, aunque pequeña, contó con una gran carga simbólica de los dispositivos retóricos expuestos en la segunda parte y que de alguna u otra manera revelaron una característica (que podría estar ausente en las otras colectividades políticas): una coherencia en el discurso del Centro Democrático durante cinco años.

La cuarta parte ("La economía política de disposiciones afectivas detrás del triunfo del No"), es, por decir lo menos, el apartado más denso dentro del conjunto total del ensayo, toda vez que si bien los anteriores apartados podrían considerarse como una especie de análisis e interpretaciones de coyuntura (realizado a través de los elementos que para esto se requieren: identificación de actores, discursos, estrategias, correlaciones de fuerza, etc.), el cuarto apartado en particular ofrece aproximaciones mucho más teóricas y de orden conceptual.

En este el autor advierte que el concepto de economía política de disposiciones afectivas (utilizado varias veces en la introducción pero que logra su acotación y explicación solo hasta esta cuarta parte) tiene su origen y construcción a través de dos fuentes. La primera, tomada de su libro publicado en 2015 Genocide, Geopolitics and Transicional Networks, en el que estipula que por disposiciones afectivas se entienden:

... los mecanismos suprarracionales —no necesariamente restringidos al lenguaje- que componen nuestros cuerpos y a través de los cuales se transmiten los contenidos subjetivos integrantes de los circuitos narrativos en los que ocurren nuestras acciones y reacciones, es decir, nuestras emociones. (Gómez-Suárez, 2015, p. 94)

En este orden de ideas, las disposiciones afectivas no son algo que pertenecen al cuerpo per se, sino que, por el contrario, se activan entre cuerpos como parte de un mecanismo de ejercicio de poder y pueden ser afectadas y dinamizadas por procesos políticos.

La segunda fuente para la construcción de su categoría - advierte el autor - recae en lo que Marx ha denominado como "economía política" y que para el caso de estudio en particular se refiere al "siempre cambiante conjunto de enlaces a través de los cuales un sentimiento circula, es producido y reproducido" (Gómez-Suárez, 2015, p. 95).

De esta manera, Gómez-Suárez advierte que la economía política de disposiciones afectivas es aquella que permite la producción y reproducción de sentimientos en una coyuntura particular, y que una vez alguno de estos mecanismos (simpatía, ${ }^{4}$ antipatía, ${ }^{5}$ indiferencia ${ }^{6}$ y olvido ${ }^{7}$ ) es colonizado, se genera una polarización entre ellos, trayendo consigo una "polarización de la economía política de disposiciones afectivas" (Gómez-Suárez, 2015, p.95) y desde la cual se podría explicar los resultados del comportamiento del conjunto de la sociedad colombiana en el marco del plebiscito refrendatorio en tanto "Uribe había logrado transmitir e instalar cuatro emociones a través de

\footnotetext{
4 “... mecanismo que nos permite sentir lo mismo que otros seres humanos y, de allí, la colectivización de emociones" (Gómez-Suárez, 2015, p. 96).

5 “... mecanismo a través del cual se transmiten emociones opuestas entre individuos o grupos” (Gómez-Suárez, 2015, p. 97).

6 “... disposición afectiva que crea un corto circuito en la colectivización de emociones” (Gómez-Suárez, 2015, p.97).

7 “... capacidad de olvidar la experiencia de una emoción transmitida”
} (Gómez-Suárez, 2015, p. 98). 
la radicalización de la simpatía: rabia, decepción, indignación y miedo" (Gómez-Suárez, 2016, p. 103).

De este apartado, podría surgirle al lector la inquietud de por qué Uribe tiende a gozar de simpatía hoy día para que dentro de un conjunto de la sociedad sus mecanismos, acciones y discursos sean bien recibidos y vistos con buenos ojos. Aquí podría encontrarse una tensión dentro del texto reseñado; por una parte, la invitación del autor según la cual, "Para el bien del país, es fundamental que Uribe revise sus acciones y las emociones que lo llevaron a actuar así" (Gómez-Suárez, 2016, p. 106), pero por otra parte, la posición de un lector desprevenido para quien le queda la duda de por qué las otras fuerzas, e incluso, los promotores del no fueron incapaces de conectar emocionalmente a la gente dentro de la naturaleza de la propuesta, esto es, la promoción de la capacidad del perdón, de la reconciliación y, en definitiva, la apuesta hacia lo que hasta el momento, nos ha sido desconocido: pensar un modelo diferente de sociedad.

Reflexiones con (pro)vocación de futuro

En la última parte del ensayo ("La repolitización de los indiferentes y el desmonte de la banalización de la política") se plantea un conjunto de reflexiones y consideraciones de orden descriptivo y propositivo; dentro del orden descriptivo, el autor presenta un balance satisfactorio sobre el accionar posplebiscito ${ }^{8} \mathrm{y}$ el conjunto de iniciativas que dieron muestra de una ciudadanía activa y que permitió, de alguna u otra manera, que se avanzara en la dinámica de la consolidación del proceso de paz. Así, se destacan la "marcha del silencio" del 5 de octubre de 2016, iniciativa que si bien contó con la aprobación de algunos sectores de la sociedad, no dejó de recibir críticas en tanto se hizo alusión a que, efectivamente, los jóvenes se manifestaron, pero lo hicieron tarde, su real participación se esperaba justo tres días antes y en las urnas. La "marcha de las flores" del 12 de octubre de 2016 y las asambleas de la iniciativa \#PazALaCalle son, entre otras, experiencias destacadas por el autor como muestra de un dinamismo de una masa crítica preocupada por el futuro del país.

Dentro del orden propositivo, Gómez-Suárez hace una invitación a algunas de las audiencias hacia las que están dirigidas sus reflexiones. En primer lugar, para que las personas apáticas a toda la situación descrita, analizada y ahora diagnosticada, erradiquen "la manipulación emocional a la que es sometida por algunos de sus dirigentes" (Gómez-Suárez, 2016, p.115) y de esta manera se evite el estancamiento de la polarización emocional ya sembrada desde 2012; también vaticina que "La solución a los problemas del país no está en que los del No y los del Sí se abracen, como muchos sugieren" (Gómez-Suárez, 2016, p. 118), sino que las personas y organizaciones articuladas en pro de la paz continúen trabajando para terminar con la banalización de la política colombiana. Para el conjunto de la sociedad, se extiende una invitación para transformar el maniqueísmo cultivado desde el uribismo a partir de frases vacías en su contenido, pero cargadas de emocionalidad que es, en últimas, lo que dinamiza y moviliza colectividades. Frente al uribismo, extiende un llamado para la cimentación de la autocrítica y la corresponsabilidad en materia de construcción de paz. Por último, hace una invitación a los jóvenes para que construyan un marco de referencia emocional (que refleje a su vez inteligencia emocional y un proyecto de país diferente) en el que prime la alimentación de tres emociones fundamentales: calma, templanza y ecuanimidad.

No podría señalar, como suele ser habitual al momento de construir reseñas, que el texto aquí descrito se presenta como de obligada consulta, pero sí como referente o fuente primaria para quienes se encuentren interesados en profundizar en el tema. Por supuesto, futuras líneas de reflexión estarían trazadas por interrogantes como: ¿cuáles fueron —si los hubo- los dispositivos retóricos del sí y por qué no fueron efectivos? ¿Por qué se continúa gobernando bajo un enfoque de Realpolitik en que en lugar de procurar diálogos constructivos se aventura, por el contrario, a desconocer el principio básico de negociación y resolución de conflictos (esto es, la aceptación del otro como un otro legítimo en la convivencia)? Y ¿cómo trabajar en el fortalecimiento, cambio y redireccionamiento de la cultura política de manera que la gente no continúe siendo indiferente hacia la política? El diálogo queda abierto.

\section{Referencias}

De Miera, A. (2017). Sociedad civily paz. Reflexiones en torno al plebiscito del 2 de octubre de 2016. Colombia: Aurora.

Gómez-Suarez, A. (2015). Genocide, Geopolitics and Transnational Networks. Londres: Routledge.

Gómez-Suarez, A. (2016). El triunfo del No: la paradoja emocional detrás del plebiscito. Bogotá D.C.: Ícono.

Jaramillo, J. (2014). Pasados y presentes de la violencia en Colombia. Estudio sobre las Comisiones de Investigación (1958-2011). Bogotá D.C.: Pontificia Universidad Javeriana.

Rodeemos el Diálogo (RED). Recuperado de http://www.rodeemoseldialogo.org

8 Dentro del conjunto de la sociedad, para dar cuenta de la desalentadora situación de los resultados por quienes apoyaron el sí, se empezó a hablar, jocosamente, de la "plebitusa". 